\title{
Histo-diagnostic and Surgical Approach for Adenoid Cystic Carcinoma on Postero-lateral Border of Tongue - a rare case report
}

\author{
Dr Shally Khanna ${ }^{1}$, Dr Narendra Nath Singh $^{2}$, Dr Gadiputi Sreedhar ${ }^{3}$, \\ Dr Anupam Purwar ${ }^{4}$ \\ ${ }^{I}$ (Assistant Professor, Department of Oral Pathology and Microbiology, Purvanchal Institute of Dental \\ Sciences, India) \\ ${ }_{2}^{2}$ (Professor and Head, Department of Oral Pathology and Microbiology, Kothiwal Dental College and \\ Research Centre, India) \\ ${ }_{3}^{3}$ Professor, Department of Oral Pathology and Microbiology, Kothiwal Dental College and Research Centre, \\ India) \\ ${ }^{4}$ (Assistant Professor, Department of Prosthodontics, Purvanchal Institute of Dental Sciences, India)
}

\begin{abstract}
Adenoid cystic carcinoma is a rare malignant salivary gland neoplasm, most commonly occurring on palate, while rarely to find in tongue. It is slowly growing tumor, being asymptomatic for long period of time. It usually present with swelling and pain at the time of diagnosis. Histopathologically, it present as three variants namely, tubular, cribriform and solid patterns of ductal and myoepithelial cells, with solid pattern being the most aggressive one. The tumor is very unpredictable, rarely showing regional spread but has higher chances of recurrence and distant metastases to lungs and bones. Hence, aggressive surgical excision with adjunct radiotherapy is the required treatment modality.
\end{abstract}

Keywords: Adenoid cystic carcinoma, Osteotomy, Glossectomy

\section{INTRODUCTION}

The first microscopic description of adenoid cystic carcinoma appeared in an 1853 paper by Robin, Lorain and Laboulbene. They used the term tumeur heteradenique, implying that it was a glandular neoplasm arising in a nonglandular area. Billroth proposed the term zylindrome (cylindroma) in 1859, because it forms a cross between connective tissue and epithelial elements which make formations that are similar in appearance to cylindromatosis. Later, in 1930 Spies used the term adenoid cystic carcinoma, stating the malignant nature of this neoplasm and avoids confusion with the benign cutaneous appendage tumor known as cylindroma. However, term adenoid cystic carcinoma is still somewhat misleading since, the tumor is not cystic $[1,2,3]$.

It was the major work of Foote and Frazell, who proposed an accepted classification of salivary gland tumors. They described that adenoid cystic tumors were located in the major and minor salivary glands. These are usually small with an incomplete capsule, variations in histology and had a propensity to perineural spread. Foote and Frazell also suggested that the low cure rates reflected a relatively conservative surgical approach. Hence, a more radical surgical treatment could show considerable improvement [4].

Adenoid cystic carcinoma is a rare malignant tumor, representing about 1 to $2 \%$ of all head and neck neoplasms and 10 to $15 \%$ of all salivary gland neoplasms. Its most common site for minor salivary gland involvement is palate, followed by base of tongue, with a rate of $8.8 \%$. In contrast, occurrence in mobile part of tongue is only $2.9 \%$, which is very rare. Regional metastases manifest in less than $3 \%$; however, distant metastases are more frequent and involve mainly the lungs and bones, followed by brain, liver, and omentum [5].

The present paper will discuss a case of adenoid cystic carcinoma on postero-lateral border of mobile part of tongue in a 45 years old female patient. It was cribriform variant histopathologically and was managed by, partial glossectomy through mandibular body split osteotomy surgical approach.

\section{Case Report}

A 45 years old female patient reported to the Outpatient Department of Kothiwal Dental College and Research Centre, Moradabad (U.P, India). Her chief complaint was swelling on left side of back region of tongue since 2 months. History revealed that, at onset the swelling was small in size and has gradually progressed. It was associated with mild pain while speech and mastication. Patient didn't visit to any clinician and it was her first visit to the outpatient department for the same problem.

Extra-oral examination revealed no remarkable finding. Intra-orally, there was presence of solitary, well demarcated, nodular, pale pink coloured swelling on left postero-lateral border of tongue opposite to left 
molar region. At focal region swelling was slightly erythematous. Swelling was $3 \mathrm{~cm} \times 3 \mathrm{~cm}$ in its greatest dimensions (Fig 1). On palpation it was soft to firm, smooth, nodular, and tender. Provisional diagnosis of traumatic fibroma was made with squamous cell carcinoma kept as differential.

After routine haematological investigations, incisional biopsy was performed and processed for histopathological assessment. Hematoxylin and eosin stained sections revealed the presence of parakeratinized stratified squamous epithelium with underlying fibrocellular connective tissue stroma. Connective tissue stroma showed the presence of ductal and myoepithelial cells forming nests with multiple pseudolumina, which were filled with eosinophilic material, giving a swiss-cheese or cribriform appearance to the tissue. Also showed the presence of collagen fibre bundles with fibroblasts compartmenting the tumor islands, chronic inflammatory cells chiefly lymphocytes and few endothelial lined blood capillaries (Fig 2). Histopathological diagnosis of Adenoid Cystic Carcinoma (Cribriform variant) was made.

Based on the histopathological diagnosis, wide surgical excision was planned by the way of partial glossectomy. Mandibular body split osteotomy or lip splitting mandibulotomy surgical technique was used to approach the tumor. Osteotomy was carried out after intentional extraction of left mandibular canine. The two mandibular segments were then pulled apart and tongue was deflected to access the tumor (Fig 3). Wide excision of tumor including $2 \mathrm{~cm}$ margin all around the tumor was performed (Fig 4). The two mandibular segments then approximated followed by plating and soft tissue closure (Fig 5 and 6). Excised tissue was reevaluated for the confirmation of diagnosis of the tumor proper and for evaluation of the excised margins for neoplastic infiltration (Fig 7). This revealed the similar histopathological features of tumor proper, with tumor margins free from neoplastic infiltration (Fig 8). Post operative follow up was done for two weeks which revealed signs of healing of excised margins. Further follow up was not maintained, due to patient's uncooperation and unavailability.

\section{DISCUSSION}

World Health Organization in 2005 defined adenoid cystic carcinoma as "A basaloid tumor consisting of epithelial and myoepithelial cells in various morphological configurations, including tubular, cribriform and solid patterns. It has a relentless clinical course and usually a fatal outcome" [6].

Foote and Frazell in 1953 suggested that both ducts and acini are the progenitors of adenoid cystic carcinoma. Hubner et al., in 1971 mentioned that adenoid cystic carcinoma originates from the myoepithelial cells. In addition to myofibrils, it contains large amount of rough endoplasmic reticulum, indicating an intensive synthesis of proteins. The acellular hyaline material is the product of myoepithelium and not of fibroblasts. Later, Anand P et al., in 1986 conducted an ultrastructural study and identified myoepithelial cells in 9 of 12 cases of adenoid cystic carcinoma. The extent of myoepithelial cell presence varied from one tumor to another. They were commonly associated with the acinar-intercalated units and tubular-ductal structures, but not with the solid areas of tumors. Thus, he stated that myoepithelial cells were from two different sources, i.e., the original acinar-intercalated ducts and the proliferation and cytodifferentiation of pluripotential reserve/stem cells [1,7].

Jun Cheng et al., in 1992 used immunohistochemical stains for basement membrane proteins. They showed that tumor cells proliferate in strands in which every cell is able to make contact with the basement membrane. These strands grow in various directions and contact each other. The invaginated and enclosed stroma is sometimes isolated from the outer stroma. The extracellular matrix enclosed by tumor cells, namely a pseudocyst, is formed by secretion and deposition of basement membrane like material by the tumor cells [7].

Most adenoid cystic carcinomas arise in the minor salivary glands (60\%). In oral cavity the common site of involvement are palate, buccal region, maxilla, retromolar region and lips [8]. One of the least frequent sites of occurrence for adenoid cystic carcinoma is the mobile part of tongue. Several authors have reported an incidence of only approximately 3\% of adenoid cystic carcinoma cases occurring on mobile part of tongue. Similar, site of presentation have been found in the present case. It has been reported that in this location, 75\% cases are in stage $\mathrm{T} 3$ or $\mathrm{T} 4$ at the time of diagnosis [9]. It may be asymptomatic for a long period and then identified by the patient as a "lump in the tongue", as it was seen in the case described. Also, it may present as a deep pain in the tongue or, rarely, with paresis of one side. Such symptoms should alert the clinician to consider this tumor in the differential diagnosis of unexplained deep tongue pain in adults [10]. Lymphatic spread being very rare, lymphadenopathy is seldom encountered, as we have seen. Lymph nodes, however, in very extensive cases, may be involved by direct extension (Allen and Marsh 1970). In long standing cases distant metastasis occurs via the blood stream to the lungs and bones. Most cases of metastatic adenoid cystic carcinoma remain asymptomatic for a long time [11].

Histologically, mixture of patterns is common in adenoid cystic carcinoma. Classification is made according to the predominant pattern. If a tumor has more than $30 \%$ of the solid pattern, it is classified as the solid variant, indicating its more aggressive behaviour. The tubular pattern (well differentiated or grade I) is characterized by slender tubules, solid cords, and glandular structures infiltrating a well-hyalinized background. Central lumina are lined with small cuboidal or columnar epithelial cells surrounded by abluminal myoepithelial 
cells. The most commonly encountered cribriform pattern (moderately differentiated or grade II) is characterized by invasive tumor islands with multiple holes (pseudocysts or pseudolumina) punched out in a "Swiss cheese" or sievelike pattern. The pseudocysts contain a rind of dense pink basement membrane material and the central blue mucopolysaccharides. True lumina are scattered between the pseudocysts and are surrounded by myoepithelial cells. Similar histological picture was seen in the present case. The solid pattern (poorly differentiated or grade III) consists of large islands of carcinoma composed predominantly of myoepithelial cells with infrequent true lumina, lined with cuboidal epithelial cells, with only occasional pseudocysts. Stroma is fibrous and extensive hyalinization can take place. Perineural spread is a feature common to all patterns, however in present case it wasn't so. Mitotic figures and apoptotic cells are occasionally present in intermediate-grade tumors and are common to high-grade or solid pattern. Necrosis is seen, usually only in the solid pattern, often centrally located within cell nests, imparting a comedo appearance [10,12].

Surgery is the cornerstone of treatment, generally requiring excision with the widest possible margins. This is because the tumor cells extend well beyond the clinical or radiographic margins and that the tumor undergoes not only perineural invasion but perineural spread. Surgery may be performed as partial glossectomy, hemiglossectomy, total glossectomy with/without preservation of the larynx, or total glossectomy with/without reconstruction if the case merits it $[9,10,13]$. Large tumors on lateral border of the tongue or those involving or extending onto the floor of the mouth may occasionally be ressected transorally with a separate neck dissection approach, but most require a lip-splitting mandibulotomy approach [14]. We performed partial glossectomy using the lip-splitting mandibulotomy approach for wide excision of tumor in the present case. Although local recurrence appears to be decreased, a survival benefit has not been demonstrated. Pathologic findings correlated with local recurrence rates, and positive resection margins were significantly associated with an increased risk of local recurrence. For lesions in the tongue, there is a greater reliance on radiotherapy. Usually, a local excision of accessible tumor is followed by high-dose radiotherapy (7,000 cGy to 8,000 cGy). In patients who receive postoperative radiation therapy, an improved outcome is observed with radical surgery compared with biopsy alone. Primary radiotherapy is recommended, for patients who refuse surgery or who are suffering from an inoperable/unresectable tumour. Whether neutron radiotherapy is more efficacious than conventional low linear energy transfer radiotherapy for patients advanced disease remains unclear. Chemotherapy currently is seeking a role in the management of advanced and metastatic salivary gland tumors, however its role remains investigational $[4,10,15,16]$. Data from a combined series of over 800 cases of adenoid cystic carcinoma from all sites shows survival rates of $75 \%$ at 5 years, $40 \%$ at 10 years, $25 \%$ at 15 years, and $20 \%$ at 20 years. The best results appear to be obtained by using a combination of radical surgery and postoperative radiation therapy, however usefulness of chemotherapy remains still to be proven $[2,10]$.

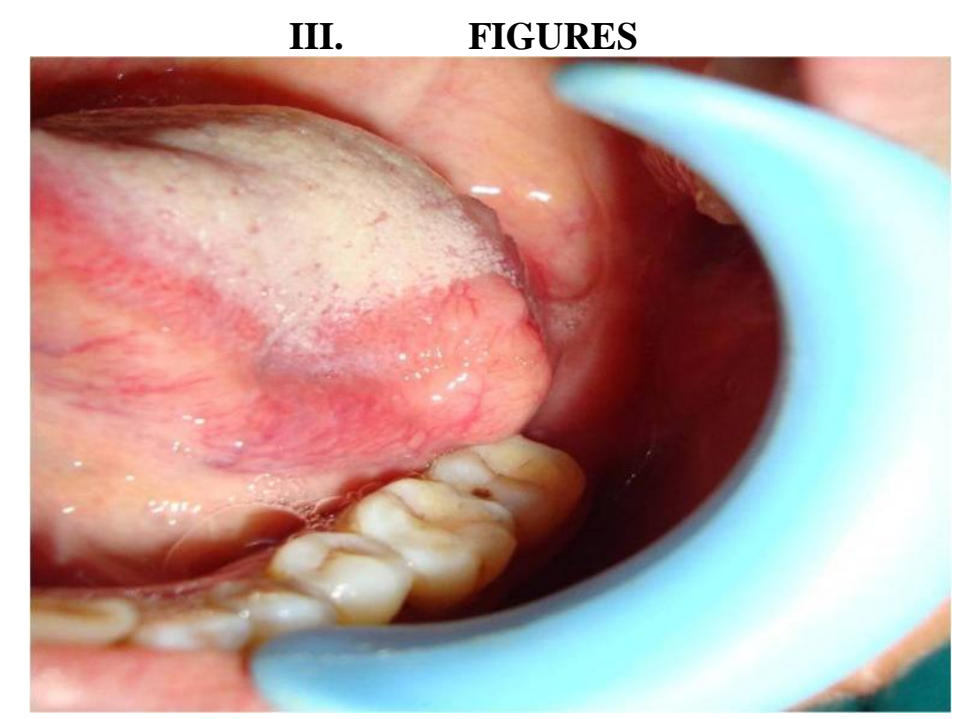

Fig 1-Clinical photograph showing swelling on left postero-lateral border of tongue. 


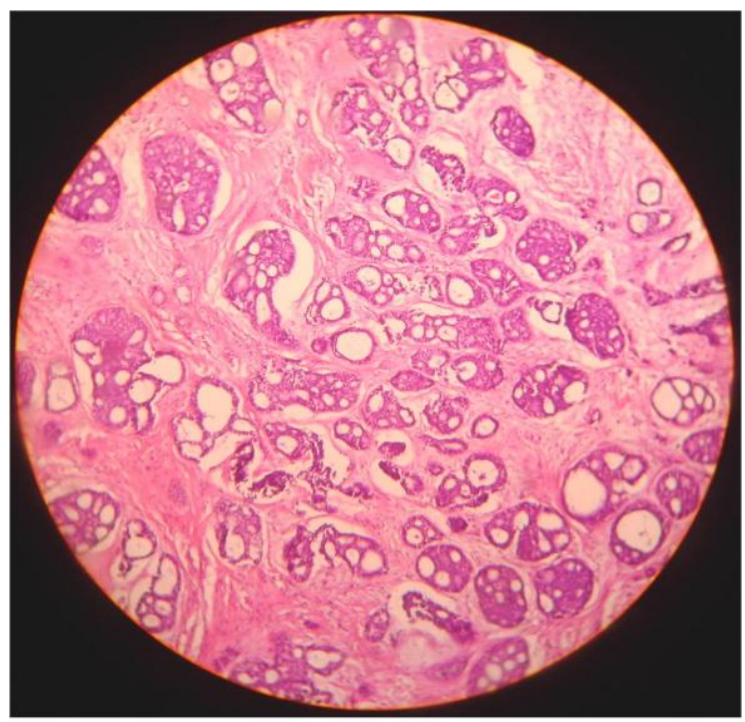

Fig 2-Photomicrograph showing cribriform pattern - adenoid cystic carcinoma (H \& E, x100).

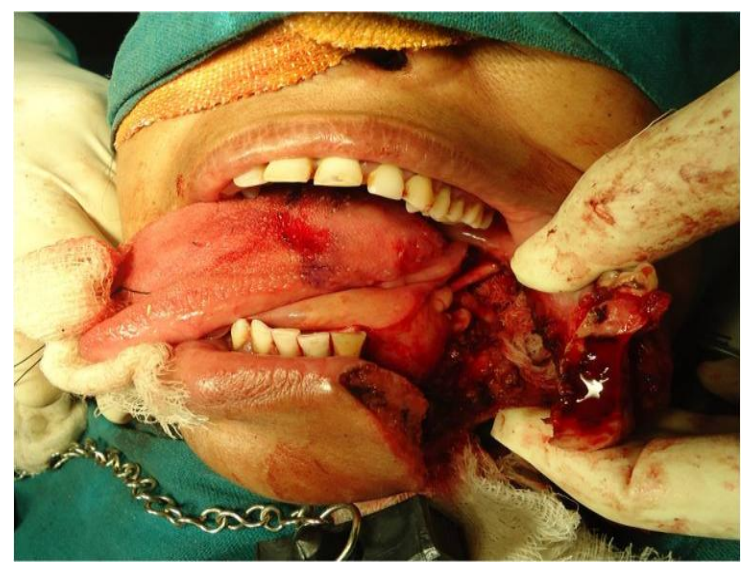

Fig 3- Mandibular body split osteotomy in 33 region.

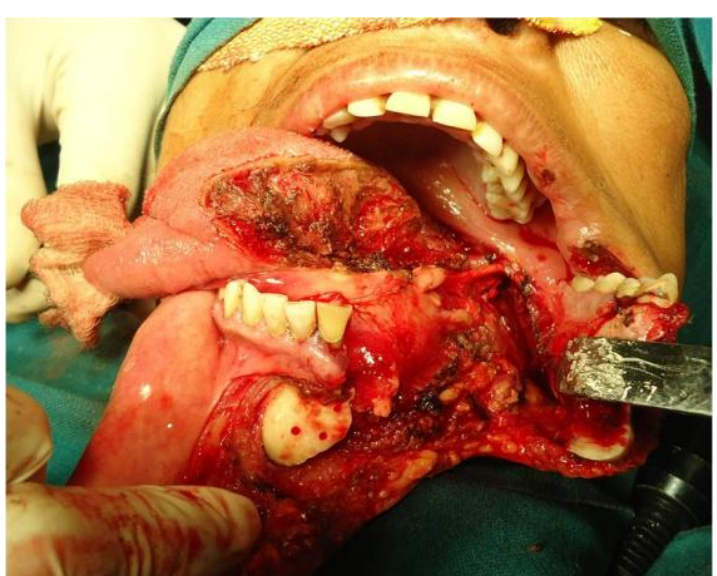

Fig 4-Wide excision of tumor mass by partial glosectomy. 
Histo-diagnostic and Surgical Approach for Adenoid Cystic Carcinoma on ...

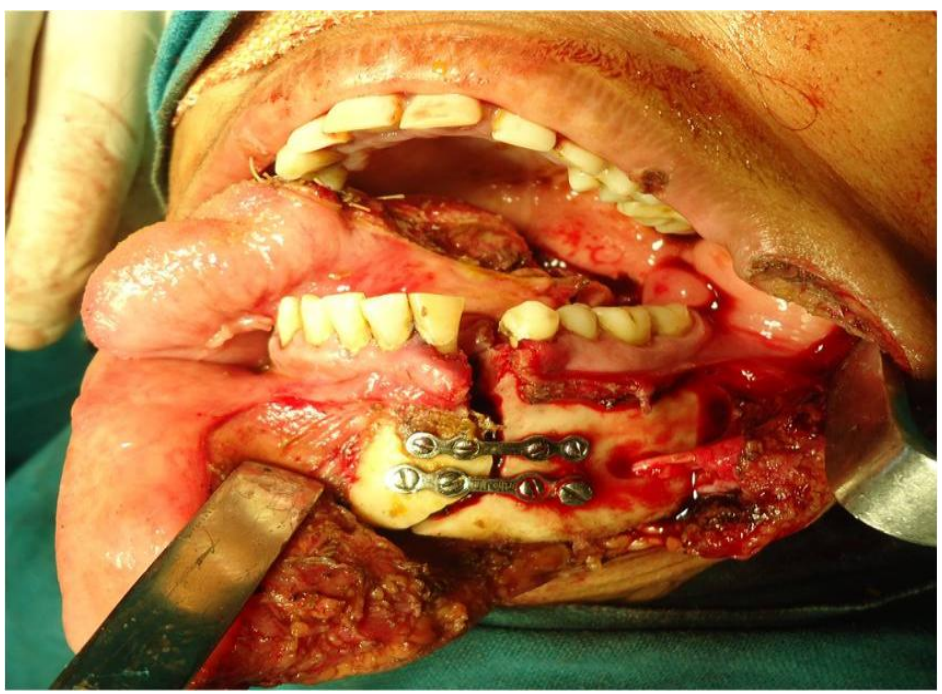

Fig 5- Plating of approximated mandibular segments.

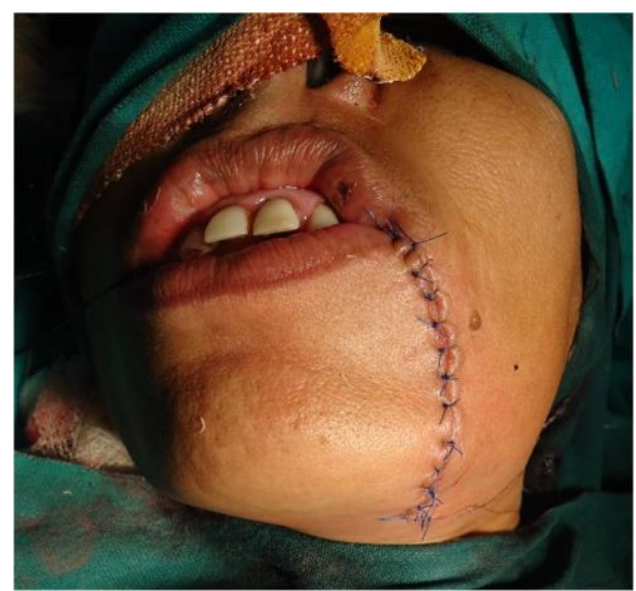

Fig 6- Soft tissue closure.

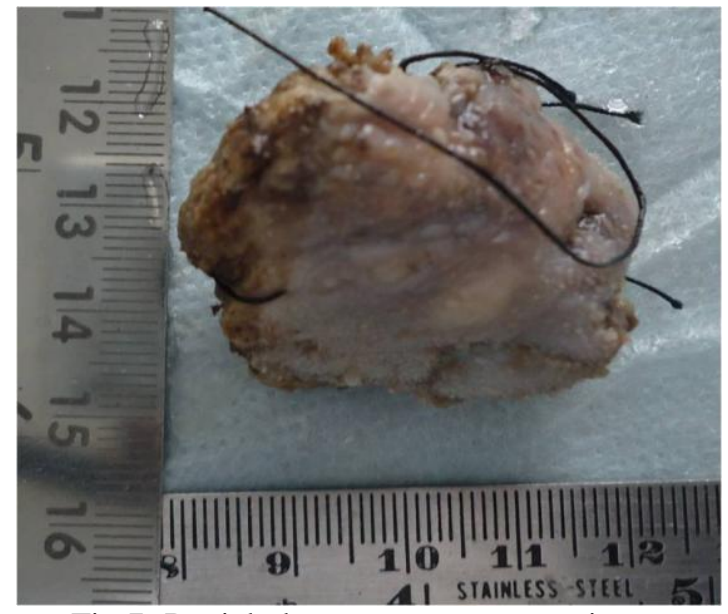

Fig 7- Partial glossectomy gross specimen. 


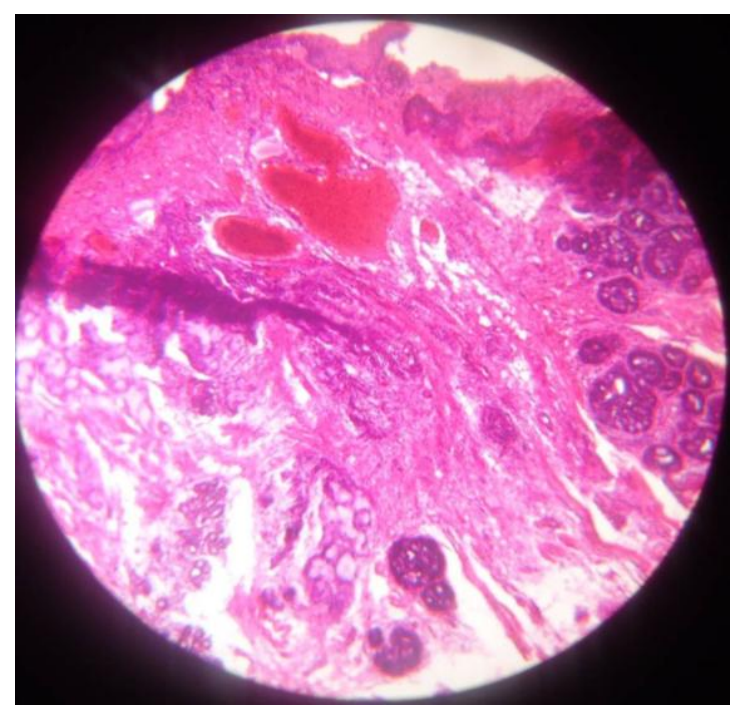

Fig 8- Photomicrograph showing tumor margin free from neoplastic infiltration (H \& E, x100).

\section{CONCLUSION}

Adenoid cystic carcinoma, a minor salivary gland neoplasm rarely involve mobile portion of tongue leading to swelling with pain. Due to the possibility of perineural spread of this tumor, surgery with wide excision of margins is the foremost step in treatment. Adjunct to surgical excision radiotherapy and chemotherapy can also be used for advanced cases. In the present case lip splitting mandibulotomy proved to be a convenient approach for partial glossectomy.

\section{Acknowledgements}

We are thankful to Dr. Sanjay Singh, Dr. Ashish Sharma, Dr. Manpreet Singh, and Dr. Ramankant Sinha for their substantial contribution in diagnosis and treatment of the case.

\section{REFERENCES}

[1]. Barnes L: Surgical pathology of the head and neck, (New York: Marcel Dekker, 2001).

[2]. Myers E. N and Ferris R. L: Salivary gland disorders (New York: Springer, 2007).

[3]. Rodriguez N. M, Berrocal I. L, Alonso L.R, Irimia O. A, Gonzalez J. M. M. Epidemiology and treatment of adenoid cystic carcinoma of the minor salivary glands: A meta-analytic study. Med Oral Patol Oral Cir Bucal 2011; 16(7):884-9.

[4]. Bradley P. J. Adenoid cystic carcinoma of the head and neck: a review. Curr Opin Otolaryngol Head Neck Surg 2004;12:127-132.

[5]. Ortiz K. L, Luna T. C, Gomez A. H, Valdez A. M. C. Macroglossia caused by adenoid cystic carcinoma - case report. Med Oral Patol Oral Cir Bucal 2008;13(6):395-7.

[6]. Barrett W and Speight PM. Perineural invasion in adenoid cystic carcinoma of the salivary glands: a valid prognostic indicator? Oral Oncology 2009;45:936-40.

[7]. Rani Hamsa PR, Anu Priya S, Arun Priya S, Thilaga Rani PR. Adenoid cystic carcinoma: a case report and review on its histogenesis and morphogenesis. International Journal of Oral \& Maxillofacial Pathology. 2012;3(2):76-80.

[8]. Shankar V. N, Prakash R, Sumalatha M. N, Shankar A. Adenoid cystic carcinoma of tongue. International Journal of Academic Research 2011;3(4):580-582.

[9]. Ortiz K. L, Luna T. C, Valdez A. M. C, Taylor A. M, Gomez A. H, Valencia V. V. Adenoid cystic carcinoma of the tongue clinicopathological study and survival analysis. Head \& Neck Oncology 2009;1(15).

[10]. Marx R. E and Stern D: Oral and maxillofacial pathology, (Hong Kong: Quintessence Publishing Co, 2003).

[11]. Dutta N. N, Baruah R, Das L. Adenoid cystic carcinoma - clinical presentation and cytological diagnosis. Indian Journal of Otolaryngology and Head and Neck Surgery 2002;54(1):62-64.

[12]. Gnepp D.R: Diagnostic surgical pathology of head and neck, (Philadelphia: WB Saunders Co, 2009).

[13]. Mahajan A, Kulkarni M, Parekh M, Khan M, Shah A, Gabhane M. Adenoid cystic carcinoma of hard palate: a case report. Oral \& Maxillofacial Pathology Journal 20011;2(1):127-131.

[14]. Montgomery P. Q, Evans P. H. R, Gullane P. J: Principles and practice of head and neck surgery and oncology, (London: Informa Healthcare, 2009).

[15]. Mendenhall W. M, Morris C. G, Amdur R. J, Werning J. W, Hinerman R. W, Villaret D. B. Radiotherapy alone or combined with surgery for adenoid cystic carcinoma of the head and neck. Head \& Neck 2004 February 154-162.

[16]. Licitra L, Grandi C, Prott F. J, Schornagel J. H, Bruzzi P, Molinari R. Major and minor salivary glands tumours. Critical Reviews in Oncology/Hematology 2003;45:215-225. 